LA W RENCE LIVERMORE N A T IO N A L LABORATORY
The Effect of Hydrogen and Fluorine Coadsorption on the Piezoelectric Properties of Graphene

M. T. Ong, K. N. Duerloo, E. J. Reed

November 13, 2012

Journal of Physical Chemistry C 
This document was prepared as an account of work sponsored by an agency of the United States government. Neither the United States government nor Lawrence Livermore National Security, LLC, nor any of their employees makes any warranty, expressed or implied, or assumes any legal liability or responsibility for the accuracy, completeness, or usefulness of any information, apparatus, product, or process disclosed, or represents that its use would not infringe privately owned rights. Reference herein to any specific commercial product, process, or service by trade name, trademark, manufacturer, or otherwise does not necessarily constitute or imply its endorsement, recommendation, or favoring by the United States government or Lawrence Livermore National Security, LLC. The views and opinions of authors expressed herein do not necessarily state or reflect those of the United States government or Lawrence Livermore National Security, LLC, and shall not be used for advertising or product endorsement purposes. 


\title{
The Effect of Hydrogen and Fluorine Coadsorption on the Piezoelectric Properties of Graphene
}

\author{
Mitchell T. Ong, ${ }^{1,2}$ Karel-Alexander N. Duerloo ${ }^{2}$ and Evan J. Reed ${ }^{2}$ \\ ${ }^{1}$ Condensed Matter and Materials Division, Physical and Life Sciences Directorate, Lawrence Livermore \\ National Laboratory, Livermore, CA 94550, USA \\ ${ }^{2}$ Department of Materials Science and Engineering, Stanford University, Stanford, CA 94305, USA
}

\begin{abstract}
:
Motivated by a search for electromechanical coupling in monolayer materials, we study graphene chemically modified by hydrogen adsorbed on one side and fluorine adsorbed on the other side. Such adsorption under experimental conditions can potentially lead to a variety of configurations of atoms on the surface. We perform an exhaustive evaluation of candidate configurations for two stoichiometries, $\mathrm{C}_{2} \mathrm{HF}$ and $\mathrm{C}_{4} \mathrm{HF}$, and examine their electromechanical properties using density functional theory. While all configurations exhibit an $e_{31}$ piezoelectric effect, the lowest energy configuration additionally exhibits an $e_{11}$ effect. Therefore, both $e_{31}$ and $e_{11}$ piezoelectricity can potentially be engineered into nonpiezoelectric monolayer graphene, providing an avenue for monolithic integration of electronic and electromechanical devices in graphene monolayers for resonators, sensors, and nanoelectromechanical systems (NEMS).
\end{abstract}

KEYWORDS: graphene, piezoelectricity, two-dimensional materials, density functional theory, nanoelectromechanical systems (NEMS). 


\section{INTRODUCTION}

Among the greatest challenges in harnessing the power of nanotechnology is achieving dynamic control of mechanical, chemical and electronic properties of nanoscale devices. Many devices stand to benefit from such control including nanosized actuators, ${ }^{1-2}$ motors, ${ }^{3}$ robots, ${ }^{4}$ locomotive devices, ${ }^{5}$ and low-powered electronics using graphene nanoelectromechanical switches. ${ }^{6}$ Conversely, endowing nanoscale devices with a robust form of in situ mechanical sensory capabilities is a challenge that is closely linked to that of achieving dynamic control. One avenue that currently exists to tackle both challenges is to employ piezoelectric materials. For instance, in a set of emerging technologies termed (nano)piezotronics, piezoelectric materials are used to make electromechanical transistors, diodes and sensors. ${ }^{7}$ Piezoelectricity is traditionally thought to be an intrinsic feature of some materials exhibiting a nonzero first-order coupling between mechanical (e.g., stress or strain) and electrical (e.g., electrical polarization or macroscopic electric field) state properties. Piezoelectricity plays a crucial role in a variety of devices such as transducers, ${ }^{8}$ nanoscale generators, ${ }^{9}$ and sensors. ${ }^{10}$ Piezoelectric materials have two key defining characteristics: (1) they can sustain a macroscopic electric field by being electrically insulating in the field direction, and (2) they belong to a non-centrosymmetric point group $^{11}$ (i.e., the crystal does not possess a point through which a spatial inversion leaves the entire structure invariant). The non-centrosymmetry requirement severely limits the number of materials that can be piezoelectric. Until recently, piezoelectricity has been primarily known as a bulk phenomenon exhibited by 3D crystals. However, there is no formal reason why the centrosymmetry argument does not apply in the two-dimensional subspace of atomically thin materials. Sparked by the isolation of graphene in 2004 by mechanical exfoliation, ${ }^{12}$ there is an explosively growing interest in using two-dimensional monolayer materials in the next 
generation of NEMS and electronic applications, naturally raising the question of whether these monolayer materials can be used in a piezoelectric context. In prior work, we have shown that many commonly studied atomically thin crystals (BN, $\mathrm{MoS}_{2}, \mathrm{MoSe}_{2}, \mathrm{WS}_{2}$, and others) intrinsically have a strong piezoelectric effect. ${ }^{13}$

In this work, we examine the piezoelectric potential of graphene, the most widely studied monolayer material. Graphene is an atomically thin layer of carbon atoms arranged in a honeycomb structure with many unique properties. Pristine, isolated graphene has high carrier mobility ${ }^{14}$ and is a zero band gap semiconductor where the valence and conduction bands are nearly conical when they intersect at a point that coincides with the Fermi energy. In addition to being an exceedingly thin material, it is also one of the most elastic and can withstand strains in excess of $10 \%$ before failure. ${ }^{15}$ Furthermore, it has been shown experimentally that graphene's electronic properties can be modified by application of an electric field ${ }^{16}$ or mechanical strain. ${ }^{17-}$ ${ }^{19}$ Current applications of graphene include field-effect transistors (FETs), ${ }^{20}$ impermeable membranes used to trap gases, ${ }^{21}$ ultrafast photodetectors, ${ }^{22}$ and nanomechanical resonators. ${ }^{23}$

Besides these exceptional electronic properties, however, graphene cannot intrinsically exhibit piezoelectric properties because it belongs to a centrosymmetric point group (6/mmm or $\mathrm{D}_{6 \mathrm{~h}}$ ). We have previously discovered that an $e_{31}$ piezoelectric response (i.e., one relating in-plane strain to electrical polarization normal to the plane) can be engineered into graphene by depositing adatoms on a single side of the graphene monolayer. ${ }^{24} \mathrm{We}$ find that, in these structures, the magnitude of the piezoelectric effect is on the same order of magnitude as that of known bulk wurtzite piezoelectrics. The demonstrated experimental ability ${ }^{25-27}$ to spatially pattern adsorbates on graphene's surface could lead to devices that utilize this piezoelectric effect in applications where strain controls local electrical, optical, and chemical properties. 
However, adatom adsorption is not the only way one could imagine breaking inversion symmetry in graphene. For example, others have performed calculations showing that an inplane $\left(e_{11}\right)$ piezoelectric effect can be induced by introducing carefully defined triangular holes in monolayer graphene, without adatom adsorption. ${ }^{28}$ In this work, we seek to determine the potential for engineering an $e_{11}$ in-plane piezoelectric effect using the standard adatom chemical deposition techniques, which have been widely developed and utilized for monolayer graphene.

The deposition of adatoms on graphene has been studied experimentally ${ }^{29-31}$ and theoretically ${ }^{32-38}$ with some studies focused on adsorption of either hydrogen or fluorine atoms on both sides (graphane and fluorographene) ${ }^{26,39-40}$ Adsorption of $\mathrm{H}$ on a single side and $\mathrm{F}$ on a single side have also been experimentally realized and shown to be stable under ambient conditions when graphene is bound to a substrate. ${ }^{26,39,41}$ Both calculations $^{42}$ and experiments ${ }^{21}$ have shown that graphene can be impermeable to atom diffusion through the sheet even in the presence of defects. We are not aware of experiments to date in this rapidly developing area where atoms of different types are coadsorbed on opposite sides of graphene. However, our calculations suggest that it may be possible to extend the experimental machinery ${ }^{26,41}$ used for single-sided adsorption to co-adsorb fluorine atoms on one side and hydrogen atoms on the other. Previous density functional theory (DFT) studies have examined one particular configuration $^{35,38}$ (\#1 in Figure 1) or stoichiometry ${ }^{43}\left(\mathrm{C}_{2} \mathrm{HF}\right)$ for $\mathrm{H}$ and $\mathrm{F}$ coadsorption on graphene. Unfortunately, it is experimentally difficult to control the location and stoichiometry of these atoms on graphene's surface. As a result, there are many different structures that can potentially be formed, most of which are metastable, and each one possessing different electromechanical properties. 
To elucidate the most energetically favorable adatom binding configuration, which one would expect to observe upon annealing under experimental conditions, we perform an exhaustive search among the possible configurations and stoichiometries of $\mathrm{H}$ and $\mathrm{F}$ coadsorbed on graphene. In addition, we calculate the Kohn-Sham DFT electronic band gap and piezoelectric response in graphene. We demonstrate, for the first time, that an $e_{11}$ type of piezoelectric response, in addition to the previously predicted $e_{31}$ response, can be engineered into monolayer graphene. This engineered piezoelectric effect provides an avenue for monolithic integration of electronic and electromechanical devices in graphene monolayers for resonators, sensors, and NEMS.

\section{COMPUTATIONAL DETAILS}

We use density functional theory implemented within the Quantum-ESPRESSO ab initio software package. ${ }^{44}$ Electron exchange and correlation effects are described using the spinpolarized generalized-gradient corrected Perdew-Burke-Ernzerhof (PBE) approximation. ${ }^{45}$ Ion cores are treated using ultrasoft (Vanderbilt) pseudopotentials and a nonlinear core correction is included for fluorine, ${ }^{46}$ with the $1 \mathrm{~s}$ states being treated as core states. Periodic boundary conditions were used in all three dimensions using an orthorhombic unit cell, which contains four carbon atoms and either two or four adatoms for the graphene systems. The hexagonal primitive cell was not used in order to align the direction of polarization along one of the lattice vectors (i.e., the $x$-axis). Thus, the $x$-axis is defined to be along the armchair direction and the $y$ axis corresponds to the zig-zag direction. A $20 \AA$ vacuum layer was used in the $z$-direction to prevent periodic images from interacting with each other. The electronic wavefunction is expanded in a plane wave basis set with an energy cutoff of 60 Ry and a charge density cutoff of 
500 Ry. Brillouin zone sampling was done using a Monkhorst-Pack mesh ${ }^{47}$ of $14 \times 14 \times 1$. All ionic relaxations and cell optimizations were performed using a force convergence threshold of 1 x $10^{-4}$ Ry/a 0 . A dipole correction was used to cancel out the artificial electric field due to the net electric dipole moment that arises in polar surface calculations. ${ }^{48}$

\section{RESULTS AND DISCUSSION}

For our calculations, $\mathrm{H}$ and $\mathrm{F}$ atoms are coadsorbed on opposite sides (i.e., all $\mathrm{H}$ atoms are on one side and all $\mathrm{F}$ atoms are on the other). This is the likely scenario for experiments where one side of the monolayer is exposed to hydrogen-rich and the other to fluorine-rich surroundings. This guarantees the removal of centrosymmetry, and suggests the possibility of piezoelectricity. As a starting point in our calculation, we also assume that fluorine and hydrogen covalently bind on top of a carbon atom in graphene similar to graphane ${ }^{39}$ and fluorographene. ${ }^{26,}$

${ }^{40}$ We verified the preference for top site binding by performing calculations starting with either $\mathrm{H}$ or $\mathrm{F}$ at a hollow site (middle of a carbon hexagon) and determining that this structure was unstable. In experiments, $\mathrm{H}$ and $\mathrm{F}$ can potentially bind to graphene in a variety of different configurations and stoichiometries. We consider two different stoichiometries: full coverage denoted by the formula unit $\mathrm{C}_{2} \mathrm{HF}$ and half coverage denoted by $\mathrm{C}_{4} \mathrm{HF}$. We consider all possible configurations for each stoichiometry within the constraint of a four carbon atom rectangular unit cell. The total number of such configurations can be obtained using an expression for placing 3 different types of indistinguishable objects in $N=4$ locations: $N ! /\left(n_{\mathrm{H}} ! n_{\mathrm{F}} ! n_{e} !\right)$, where $n_{\mathrm{H}}$ is the number of hydrogen atoms, $n_{\mathrm{F}}$ is the number of fluorine atoms, $n_{e}$ is the number of empty carbon sites, and $N=n_{\mathrm{H}}+n_{\mathrm{F}}+n_{e}$. This expression assumes only one adatom can occupy a carbon site. For $\mathrm{C}_{2} \mathrm{HF}$, there are a total of $4 ! /(2 ! \times 2 ! \times 0 !)=6$ possible initial configurations. For $\mathrm{C}_{4} \mathrm{HF}$, there are a 
total of $4 ! /(1 ! \times 1 ! \times 2 !)=12$ possible initial configurations. For each stoichiometry, there are only 3 unique configurations that are not symmetry-equivalent and where all like atoms bind on the same side. The cell-optimized structures for all unique configurations of $\mathrm{C}_{2} \mathrm{HF}$ and $\mathrm{C}_{4} \mathrm{HF}$ are given in Figure 1.

In Table 1, we calculate the formation, binding and relative energies for each of these configurations along with the Kohn-Sham band gap. The formation energy per atom is defined as:

$$
E_{\mathrm{f}}=\frac{1}{N_{\text {atoms }}}\left(E_{\mathrm{d}}-E_{\mathrm{g}}-\frac{N_{\mathrm{H}}}{2} E_{\mathrm{H}_{2}}-\frac{N_{\mathrm{F}}}{2} E_{\mathrm{F}_{2}}\right)
$$

and the binding energy per adatom is expressed as:

$$
E_{\mathrm{b}}=\frac{1}{N_{\text {adatoms }}}\left(E_{\mathrm{d}}-E_{\mathrm{g}}-N_{\mathrm{H}} E_{\mathrm{H}}-N_{\mathrm{F}} E_{\mathrm{F}}\right),
$$

$E_{\mathrm{d}}$ is the total energy of the optimized graphene-adatom system with energetically relaxed ion positions, $E_{\mathrm{g}}$ is the total energy of an optimized graphene 4-carbon atom rectangular unit cell, and $E_{\mathrm{X}}$ are the total energies for $\mathrm{X}=\mathrm{H}_{2}, \mathrm{~F}_{2}, \mathrm{H}$ or $\mathrm{F} . N_{\mathrm{H}}$ and $N_{\mathrm{F}}$ denote the number of $\mathrm{H}$ or $\mathrm{F}$ atoms, while $N_{\text {atoms }}$ and $N_{\text {adatoms }}$ indicate the total number of atoms or adatoms in the unit cell. The formation and binding energies are a measure of chemical stability. A negative formation energy indicates that the structure is stable with respect to molecular desorption, while a negative binding energy indicates that the structure is stable with respect to the isolated adatoms. Most of the structures in Table 1 have negative binding and formation energies (with the exception of configuration 5) and hence, are expected to be chemically stable.

We find that configuration 1 is the most energetically favorable, with hydrogen and fluorine being adsorbed on alternating carbon sites. This structure exhibits a change in point group symmetry from hexagonal $6 / \mathrm{mmm}$ to trigonal $3 \mathrm{~m}$, removing the inversion center and 
potentially introducing piezoelectricity in graphene. It has also been examined in previous DFT calculations. $^{35,38,43}$ Our calculations show it is more favorable by only $0.05 \mathrm{eV} /$ atom over the next lowest energy structure, suggesting that other metastable structures could be observed experimentally. We find that configuration 1 has a Kohn-Sham band gap of $2.61 \mathrm{eV}$, similar to the gap of $2.63 \mathrm{eV}$ that has been reported in literature ${ }^{38}$ for the same $\mathrm{C}_{2} \mathrm{HF}$ structure using the GGA approximation and PBE exchange-correlation functional. Configurations 2-4 also exhibit a gap and change the point group symmetry of graphene from $6 / \mathrm{mmm}$ to orthorhombic $\mathrm{mm} 2$ or monoclinic $m$, while configurations 5 and 6 are both monoclinic $m$, and do not exhibit a gap.

The lack of inversion symmetry and introduction of a band gap could potentially lead to a piezoelectric effect. This effect can be expressed using the full third-rank piezoelectric tensors $e_{i j k}$ and $d_{i j k}$. Their respective Maxwell relations are:

$$
\begin{gathered}
e_{i j k}=\left(\frac{\partial P_{i}}{\partial \varepsilon_{j k}}\right)_{E, T}=-\left(\frac{\partial \sigma_{j k}}{\partial E_{i}}\right)_{\varepsilon, T}, \\
d_{i j k}=\left(\frac{\partial P_{i}}{\partial \sigma_{j k}}\right)_{E, T}=\left(\frac{\partial \varepsilon_{j k}}{\partial E_{i}}\right)_{\varepsilon, T},
\end{gathered}
$$

where $P_{i}$ is the surface polarization, $E_{i}$ is the macroscopic electric field, and $\sigma_{j k}$ or $\varepsilon_{j k}$ are the stress or strain tensors, where $i, j, k \in\{1,2,3\}$, with 1,2 and 3 corresponding to $x, y$, and $z$, respectively. The point group symmetry for each structure in Figure 1 places restrictions on the magnitude and uniqueness of the piezoelectric coefficients. Nonzero piezoelectric coefficients for trigonal $3 m$, orthorhombic $m m 2$ and monoclinic $m$ are listed in Table 2. Note that Voigt notation can be employed to reduce the number of indices to two.

We calculate the $e_{11}\left(e_{111}\right.$ in tensor notation) piezoelectric coefficient using the Maxwell relation given in Equation 3 relating uniaxial in-plane strain to the change in surface polarization 
along the same axis. To compute the change in polarization, we use the geometric phase approach introduced by King-Smith and Vanderbilt. ${ }^{49-50}$ The piezoelectric $e_{11}$ coefficient can then be obtained by fitting to Equation 5:

$$
P_{1}\left(\varepsilon_{11}\right)-P_{1}(0)=e_{11} \varepsilon_{11}
$$

In Figure 2, we determine the change in polarization along the $x$-axis in response to a uniaxial strain $\varepsilon_{11}$ for configurations 1 and 4 , which are the only structures that are not metallic and are allowed to have nonzero $e_{11}$ piezoelectric coefficients by symmetry. ${ }^{11}$ For each strain state, the atomic positions are relaxed generating so-called relaxed-ion $e_{11}$ coefficients, which are calculated from the slopes of Figure 2 and listed in Table 3. The notation $e_{11,2 \mathrm{D}}$ is used in Table 3 to denote a piezoelectric coefficient for a $2 \mathrm{D}$ material where the dimensions are charge per unit length. We find that configuration 1 results in the largest piezoelectric coefficient $\left(6.30 \times 10^{-11}\right.$ $\mathrm{C} / \mathrm{m})$, while configuration 4 exhibits a much weaker piezoelectric effect $\left(7.9 \times 10^{-12} \mathrm{C} / \mathrm{m}\right)$.

To put the magnitudes of these coefficients into context, Table 3 compares the $e_{11}$ coefficients to those of monolayer boron nitride and a bulk, 3D piezoelectric material, $\alpha$-quartz. For comparison to bulk materials, we calculate an approximate $e_{11,3 \mathrm{D}}$ relaxed-ion coefficient by dividing the $e_{11,2 \mathrm{D}}$ coefficient by the graphite interlayer spacing ( $\left.3.35 \AA\right) \cdot{ }^{51}$ Comparing the $e_{11,2 \mathrm{D}}$ coefficients of configuration 1 to $2 \mathrm{D}-\mathrm{hBN}$, we find that the piezoelectric effect is larger than might be expected and only a factor of 2 less than 2D-hBN. Comparing the $e_{11,3 \mathrm{D}}$ for configuration $1\left(0.188 \mathrm{C} / \mathrm{m}^{2}\right)$ to $\alpha$-quartz $\left(0.171 \mathrm{C} / \mathrm{m}^{2}\right),{ }^{52}$ we find that it is comparable, indicating that an in-plane piezoelectric response engineered by adsorbing atoms on graphene's surface can actually be comparable to that in bulk materials used in practical applications. This piezoelectric response is also larger than the effect predicted to exist in graphene when nanoscale triangular holes are etched $\left(0.124 \mathrm{C} / \mathrm{m}^{2}\right),{ }^{28}$ indicating that adatom adsorption is a fruitful path for 
observation of $e_{11}$ piezoelectricity. Adatom adsorption also produces larger band gaps than the etching of shaped holes in graphene, enabling the application of larger amplitude fields across the material.

An estimate of the voltage across a strained sample can be made using the electrostatic potential defined by two parallel line charges separated by a length $\mathrm{L}$. The voltage across freelysuspended material can be approximated from the expression (SI):

$$
V=\frac{P}{\pi \varepsilon_{0}} \ln \left(\frac{L}{b}\right),
$$

where $P$ is the $2 \mathrm{D}$ polarization obtained from Equation 5, $L$ is the distance between line charges (taken to be 1 micron), and $b$ is the effective radius of the lines (assumed to be a lattice parameter of $2.57 \AA$ ). This approximation suggests a voltage of $0.2 \mathrm{~V}$ assuming a $1 \%$ strain across a 1 micron sample (10 $\mathrm{nm}$ change in length).

Since the lowest energy configuration is found to exhibit the largest $e_{11}$ piezoelectric effect, one might expect that an annealing process will maximize the piezoelectric properties of this material. For these calculations, we have assumed that the adatoms form a perfect crystal on graphene's surface, neglecting the possibility of multiple domains. For configuration 1, reflection through the $y z$ plane generates an equivalent energy structure but with opposite piezoelectric polarization direction. Note that these are domains of the adsorbed adatoms rather than domains in the underlying graphene. Because pristine graphene does not favor a particular piezoelectric axis, domains with uncorrelated orientations could significantly diminish the $e_{11}$ coefficient. It is possible that annealing the sample will minimize the number of domains, thus maximizing the piezoelectric effect.

Using the two-dimensional elastic stiffness tensor for trigonal $3 m$ point group symmetry, we calculate the $d_{11}$ coefficient (Equation 4 ) for configuration 1 from the following expression: 


$$
d_{11}=\frac{e_{11}}{\left(C_{11}-C_{12}\right)}
$$

To obtain the elastic coefficients, we compute the total energy per unit area for a $5 \times 5$ grid with axial in-plane strains $\left(\varepsilon_{11}\right.$ and $\left.\varepsilon_{22}\right)$ ranging from -0.01 to 0.01 in steps of 0.005 . Atomic positions are relaxed at each strain to give the relaxed-ion elastic coefficients. Planar $C_{11}, \mathrm{C}_{22}$, and $C_{12}$ coefficients are obtained by fitting the total energy to the general expression:

$$
U=\frac{1}{2} C_{11} \varepsilon_{11}^{2}+\frac{1}{2} C_{22} \varepsilon_{22}^{2}+C_{12} \varepsilon_{11} \varepsilon_{22}
$$

$C_{11}$ and $C_{22}$ are identical for trigonal structures such as configuration 1 . We calculated $C_{11}=C_{22}$ $=243 \mathrm{~N} / \mathrm{m}$ and $C_{12}=25 \mathrm{~N} / \mathrm{m}$ for configuration 1 . These coefficients satisfy the Born stability criteria $^{53}$ where the only relevant conditions for a two-dimensional trigonal lattice are $C_{11}>0$ and $C_{11}-\left|C_{12}\right|>0$. In addition, phonon modes calculated for configuration 1 show no imaginary frequencies, indicating that this structure is mechanically stable.

Using our fitted values of $C_{11}$ and $C_{12}$ for configuration 1 , we calculate a $d_{11}$ piezoelectric coefficient of $0.235 \mathrm{pm} / \mathrm{V}$, which is a factor of 3 lower than the $d_{11}$ for hexagonal boron nitride $(0.60 \mathrm{pm} / \mathrm{V})$ and an order of magnitude lower than $\alpha$-quartz $(2.31 \mathrm{pm} / \mathrm{V})$. For context, if we assume that a field of $0.1 \mathrm{~V} / \mathrm{nm}$ is applied to a 10-micron length of material, we find that this would result in a $0.002 \%$ strain or $2.4 \AA$ change in length.

The symmetry of these structures also supports a nonzero $e_{31}$ piezoelectric coefficient, which relates the in-plane strain to electrical polarization normal to the plane. Our earlier work has shown this is possible even for metallic materials ${ }^{24}$ such as configurations 5 and 6 since they are only conducting in the plane, allowing for static polarization and electric field normal to the surface. It should be noted, however, that configuration 1 is the only structure where $e_{31}=e_{32}$ because of its trigonal $3 m$ symmetry. Due to the small thickness, it is experimentally possible to 
apply electric fields as large as $0.3 \mathrm{~V} / \AA^{16}$ normal to the plane, a feature that is not shared by bulk analogues and will be very useful in nanoscale devices such as relays and FETs. In Figure 3, we show that a linear relationship exists between uniaxial strain, $\varepsilon_{11}$, and polarization normal to the graphene sheet for all six configurations in Figure 1. We determine $e_{31,2 \mathrm{D}}$ from the slope of the lines in Figure 3 and list them in Table 4. It can be seen that most of the configurations have similar values for $e_{31,2 \mathrm{D}}$ with the largest being configurations 1 and $6\left(-3.0 \times 10^{-11} \mathrm{C} / \mathrm{m}\right)$, indicating that configuration 1 exhibits both $e_{31}$ and $e_{11}$ types of piezoelectricity. These $e_{31,2 \mathrm{D}}$ coefficients are slightly lower than the effect we predicted for Li adsorbed on graphene in earlier work $\left(5.5 \times 10^{-11} \mathrm{C} / \mathrm{m}\right)$, but lie within the range of other adatoms such as $\mathrm{H}, \mathrm{F}$ and the combination of $\mathrm{Li}$ and $\mathrm{F}^{24}$

\section{CONCLUSIONS}

We have demonstrated that the arrangement of hydrogen and fluorine atoms on the surface of graphene can affect its electromechanical properties. An exhaustive search of configurations for $\mathrm{C}_{2} \mathrm{HF}$ and $\mathrm{C}_{4} \mathrm{HF}$ resulted in the identification of six unique configurations, three for $\mathrm{C}_{2} \mathrm{HF}$ and three for $\mathrm{C}_{4} \mathrm{HF}$. Of these configurations, the most energetically favorable one is where all carbon sites are occupied with alternating hydrogen atoms above the surface and fluorine atoms below the surface. Because it has the lowest energy, this configuration can potentially be obtained by using an annealing process to minimize the number of grains in the sample and maximize the piezoelectric effect. Of all the configurations studied here, only two potentially exhibit in-plane $\left(e_{11}\right)$ piezoelectricity. The lowest energy configuration exhibits both a non-zero $e_{11}$ and $e_{31}$ piezoelectric effect. For this case, the effect is comparable to an existing bulk piezoelectric material. We anticipate that this work will open up the possibility for the 
creation of new nanoelectromechanical devices such as FETs, relays and sensors, which utilize this engineered piezoelectric effect in graphene to dynamically control mechanical motion at the nanoscale level. Such piezoelectric devices could be monolithically integrated with other electronic devices on a single piece of graphene by controlling the spatial regions that are chemically modified and endowed with piezoelectricity.

\section{CORRESPONDING AUTHOR:}

*Evan Reed, Phone: (650) 723-2971. Fax: (650) 725-4034. Email: evanreed@stanford.edu.

\section{ACKNOWLEDGMENT:}

Our work was performed at Stanford University and supported in part by the U.S. Army Research Laboratory, through the Army High Performance Computing Research Center, Cooperative Agreement W911NF-07-0027. This work was also partially supported by DARPA YFA grant N66001-12-1-4236, and used resources of the National Energy Research Scientific Computing Center (NERSC), which is supported by the Office of Science of the U.S. Department of Energy under Contract No. DE-AC02-05CH11231. Some calculations were performed in part using the Stanford NNIN Computing Facility (SNCF), a member of the National Nanotechnology Infrastructure Network (NNIN), supported by the National Science Foundation (NSF). Part of this work was performed under the auspices of the U.S. Department of Energy by Lawrence Livermore National Laboratory under Contract DE-ACS2-07NA27344. The authors would also like to thank Dr. Tingting Qi for useful comments and discussion about this manuscript. 


\section{REFERENCES:}

(1) Fennimore, A. M.; Yuzvinsky, T. D.; Han, W. Q.; Fuhrer, M. S.; Cumings, J.; Zettl, A., Nature 2003, 424 (6947), 408-410.

(2) Park, S.; An, J.; Suk, J. W.; Ruoff, R. S., Small 2010, 6 (2), 210-212.

(3) Van Delden, R. A.; ter Wiel, M. K. J.; Pollard, M. M.; Vicario, J.; Koumura, N.; Feringa, B. L., Nature 2005, 437 (7063), 1337-1340.

(4) Requicha, A. A. G., Proc. IEEE 2003, 91 (11), 1922-1933.

(5) Shirai, Y.; Osgood, A. J.; Zhao, Y. M.; Kelly, K. F.; Tour, J. M., Nano Lett. 2005, 5 (11), 2330-2334.

(6) Shi, Z. W., et al., Nano Res. 2012, 5 (2), 82-87.

(7) Wang, Z. L., Adv. Mater. 2007, 19 (6), 889-892.

(8) Jaffe, H.; Berlincourt, D. A., Proc. IEEE 1965, 53 (10), 1372-1386.

(9) Wang, Z. L.; Song, J. H., Science 2006, 312 (5771), 242-246.

(10) Morten, B.; Decicco, G.; Prudenziati, M., Sens. Actuator A-Phys. 1992, 31 (1-3), 153158.

(11) Nye, J. F., Physical Properties of Crystals: Their Representation by Tensors and Matrices. Clarendon Press: Oxford, 1957.

(12) Novoselov, K. S.; Geim, A. K.; Morozov, S. V.; Jiang, D.; Zhang, Y.; Dubonos, S. V.; Grigorieva, I. V.; Firsov, A. A., Science 2004, 306 (5696), 666-669.

(13) Duerloo, K. -A. N.; Ong, M. T.; Reed, E. J., Journal of Physical Chemistry Letters 2012, 3 (19), 2871-2876.

(14) Chen, J. H.; Jang, C.; Xiao, S. D.; Ishigami, M.; Fuhrer, M. S., Nat. Nanotechnol. 2008, 3 (4), 206-209.

(15) Lee, C.; Wei, X. D.; Kysar, J. W.; Hone, J., Science 2008, 321 (5887), 385-388.

(16) Zhang, Y.; Tang, T.-T.; Girit, C.; Zhao, H.; Martin, M. C.; Zettl, A.; Crommie, M. F.; Shen, Y. R.; Feng, W., Nature 2009, 459 (7248), 820-823.

(17) Ferralis, N.; Maboudian, R.; Carraro, C., Phys. Rev. Lett. 2008, 101 (15), 156801.

(18) Kim, K. S.; Zhao, Y.; Jang, H.; Lee, S. Y.; Kim, J. M.; Kim, K. S.; Ahn, J.-H.; Kim, P.; Choi, J.-Y.; Hong, B. H., Nature 2009, 457 (7230), 706-710.

(19) Teague, M. L.; Lai, A. P.; Velasco, J.; Hughes, C. R.; Beyer, A. D.; Bockrath, M. W.; Lau, C. N.; Yeh, N. C., Nano Lett. 2009, 9 (7), 2542-2546.

(20) Oostinga, J. B.; Heersche, H. B.; Liu, X. L.; Morpurgo, A. F.; Vandersypen, L. M. K., Nat. Mater. 2008, 7 (2), 151-157.

(21) Bunch, J. S.; Verbridge, S. S.; Alden, J. S.; van der Zande, A. M.; Parpia, J. M.; Craighead, H. G.; McEuen, P. L., Nano Lett. 2008, 8 (8), 2458-2462.

(22) Xia, F.; Mueller, T.; Lin, Y.; Valdes-Garcia, A.; Avouris, P., Nat. Nanotechnol. 2009, 4 (12), 839-843.

(23) Chen, C. Y.; Rosenblatt, S.; Bolotin, K. I.; Kalb, W.; Kim, P.; Kymissis, I.; Stormer, H. L.; Heinz, T. F.; Hone, J., Nat. Nanotechnol. 2009, 4 (12), 861-867.

(27) Sessi, P.; Guest, J. R.; Bode, M.; Guisinger, N. P., Nano Lett. 2009, 9 (12), 4343-4347.

(28) Chandratre, S.; Sharma, P., Appl. Phys. Lett. 2012, 100 (2). 
(29) Chen, J. H.; Jang, C.; Adam, S.; Fuhrer, M. S.; Williams, E. D.; Ishigami, M., Nat. Phys. 2008, 4 (5), 377-381.

(30) Li, B.; Zhou, L.; Wu, D.; Peng, H. L.; Yan, K.; Zhou, Y.; Liu, Z. F., ACS Nano 2011, 5 (7), 5957-5961.

(31) Ohta, T.; Bostwick, A.; Seyller, T.; Horn, K.; Rotenberg, E., Science 2006, 313 (5789), 951-954.

(32) Boukhvalov, D. W.; Katsnelson, M. I., J. Phys.-Condes. Matter 2009, 21 (34).

(33) Chan, K. T.; Neaton, J. B.; Cohen, M. L., Phys. Rev. B 2008, 77 (23), 235430.

(34) Jin, K. H.; Choi, S. M.; Jhi, S. H., Phys. Rev. B 2010, 82 (3), 033414.

(35) Klintenberg, M.; Lebegue, S.; Katsnelson, M. I.; Eriksson, O., Phys. Rev. B 2010, 81 (8), 085433.

(36) Leenaerts, O.; Peelaers, H.; Hernandez-Nieves, A. D.; Partoens, B.; Peeters, F. M., Phys. Rev. B 2010, 82 (19).

(37) Lugo-Solis, A.; Vasiliev, I., Phys. Rev. B 2007, 76 (23), 235431.

(38) Medeiros, P. V. C.; Mascarenhas, A. J. S.; Mota, F. D.; de Castilho, C. M. C., Nanotechnology 2010, 21 (48).

(39) Elias, D. C., et al., Science 2009, 323 (5914), 610-613.

(40) Nair, R. R., et al., Small 2010, 6 (24), 2877-2884.

(41) Lee, W. H.; Suk, J. W.; Chou, H.; Lee, J. H.; Hao, Y. F.; Wu, Y. P.; Piner, R.; Aldnwande, D.; Kim, K. S.; Ruoff, R. S., Nano Lett. 2012, 12 (5), 2374-2378.

(42) Leenaerts, O.; Partoens, B.; Peeters, F. M., Appl. Phys. Lett. 2008, 93 (19).

(43) Singh, R.; Bester, G., Phys. Rev. B 2011, 84 (15).

(44) Giannozzi, P., et al., J. Phys.-Condes. Matter 2009, 21 (39), 395502.

(45) Perdew, J. P.; Burke, K.; Ernzerhof, M., Phys. Rev. Lett. 1996, 77, 3865-3868.

(46) Vanderbilt, D., Phys. Rev. B 1990, 41, 7892-7895.

(47) Monkhorst, H. J.; Pack, J. D., Phys. Rev. B 1976, 13, 5188-5192.

(48) Bengtsson, L., Phys. Rev. B 1999, 59 (19), 12301-12304.

(49) King-Smith, R. D.; Vanderbilt, D., Phys. Rev. B 1993, 47 (3), 1651-1654.

(50) Resta, R.; Vanderbilt, D., Theory of Polarization: A Modern Approach. Springer-Verlag: 2008; p 31-68.

(51) Bacon, G. E., Acta Crystallogr. 1951, 4, 558-561.

(52) Bechmann, R., Phys. Rev. 1958, 110 (5), 1060-1061.

(53) Born, M.; Huang, K., Dynamical Theory of Crystal Lattices. Clarendon Press: Oxford, 1954. 
A

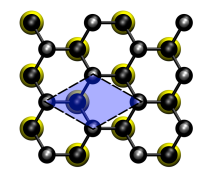

1

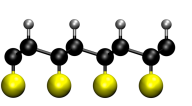

Trigonal

$3 \mathrm{~m}$

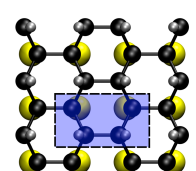

2

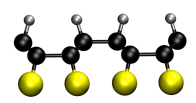

Orthorhombic

$\mathrm{mm} 2$
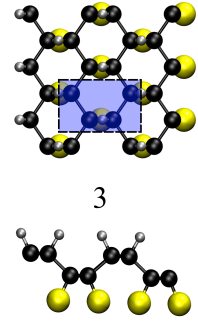

Orthorhombic

$\mathrm{mm} 2$

B
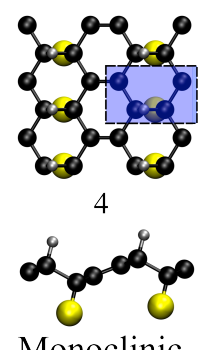

Monoclinic

$\mathrm{m}$

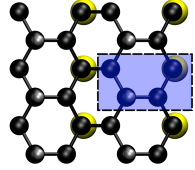

5

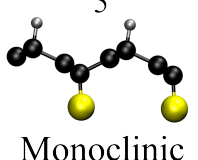

$\mathrm{m}$
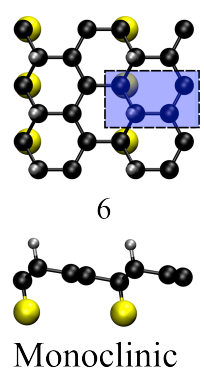

$\mathrm{m}$

Figure 1. Unique configurations for (a) $\mathrm{C}_{2} \mathrm{HF}$ and (b) $\mathrm{C}_{4} \mathrm{HF}$. Point group symmetry is also given. Primitive cells are shown in blue. Note that a hexagonal primitive cell is shown for configuration \#1, but a 4-carbon atom rectangular unit cell was used for all configurations.

Table 1. Binding, formation and energies relative to configuration \#1 for all of the unique configurations (shown in Figure 1) for $\mathrm{C}_{n} \mathrm{HF}$ where $n=2$ or 4 . Kohn-Sham band gaps are also given for each structure. Binding energies are given in eV/adatom while formation energy and energies relative to configuration \#1 are given in units of eV/atom.

\begin{tabular}{lccccc}
\hline \hline Stoichiometry & Config. \# & $\mathrm{E}_{\mathrm{f}}(\mathrm{eV} /$ atom $)$ & $\mathrm{E}_{\mathrm{b}}(\mathrm{eV} /$ adatom $)$ & $\Delta \mathrm{E}(\mathrm{eV} /$ atom $)$ & Band Gap (eV) \\
\hline $\mathrm{C}_{2} \mathrm{HF}$ & 1 & -0.43 & -2.59 & 0.00 & 2.61 \\
$\mathrm{C}_{2} \mathrm{HF}$ & 2 & -0.36 & -2.44 & 0.07 & 2.63 \\
$\mathrm{C}_{2} \mathrm{HF}$ & 3 & -0.39 & -2.49 & 0.05 & 2.44 \\
$\mathrm{C}_{4} \mathrm{HF}$ & 4 & -0.18 & -2.27 & 0.25 & 3.56 \\
$\mathrm{C}_{4} \mathrm{HF}$ & 5 & 0.21 & -1.09 & 0.64 & Metallic \\
$\mathrm{C}_{4} \mathrm{HF}$ & 6 & -0.22 & -2.40 & 0.21 & Metallic \\
\hline \hline
\end{tabular}


Table 2. Nonzero $e_{i j k}$ and $d_{i j k}$ piezoelectric coefficients for trigonal $3 m$, orthorhombic $m m 2$ and monoclinic $m$ point groups. Voigt notation is used to reduce the number of indices as indicated.

\begin{tabular}{llll}
\hline \hline & Trigonal $3 m$ & Orthorhombic $m m 2$ & Monoclinic $m$ \\
\hline$e_{i j k}$ & $e_{111}=e_{11}$ & $e_{311}=e_{31}$ & $e_{111}=e_{11}$ \\
& $e_{122}=e_{12}=-e_{11}$ & $e_{322}=e_{32}$ & $e_{122}=e_{12}$ \\
& $e_{212}=e_{26}=-e_{11}$ & & $e_{212}=e_{26}$ \\
& $e_{311}=e_{31}$ & & $e_{311}=e_{31}$ \\
& $e_{322}=e_{32}=e_{31}$ & & $e_{322}=e_{32}$ \\
& $d_{111}=d_{11}$ & & $d_{111}=d_{11}$ \\
$d_{i j k}$ & $d_{311}=d_{31}$ & $d_{122}=d_{12}$ \\
& $d_{212}=\frac{1}{2} d_{26}=-d_{11}$ & & $d_{212}=\frac{1}{2} d_{26}$ \\
& $d_{311}=d_{31}$ & & $d_{311}=d_{31}$ \\
$d_{322}$ & $=d_{32}=d_{31}$ & & $d_{322}=d_{32}$ \\
\hline \hline
\end{tabular}




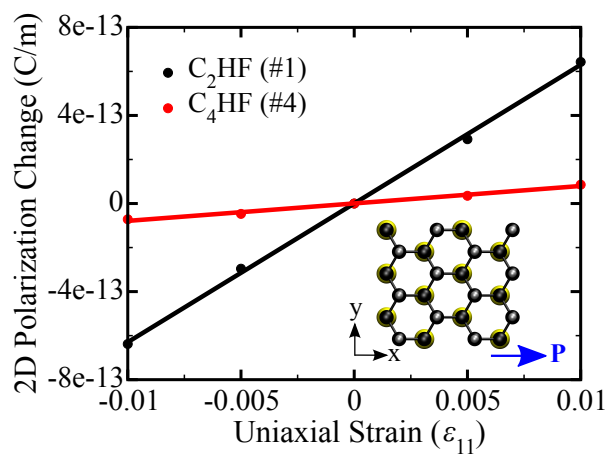

Figure 2. Applying uniaxial strain $\varepsilon_{11}$ along the $x$-direction (defined in lower right inset) of the graphene sheet induces a change in calculated polarization along the same axis for configurations 1 and 4. Atoms are relaxed under uniaxial strain along the $x$-direction to obtain the relaxed-ion coefficient. The lower right inset indicates the coordinate system and direction of polarization.

Table 3. Values for the relaxed $e_{11,2 \mathrm{D}}$ piezoelectric coefficients for configurations 1 and 4. Values for $e_{11,3 \mathrm{D}}$ were determined by dividing the $e_{11,2 \mathrm{D}}$ coefficient by the graphene interlayer spacing for each doped-graphene structure. The $d_{11}$ coefficient for configuration 1 is obtained from the relationship between $e_{11,2 \mathrm{D}}$ and the elastic tensor. These values are compared to calculated values for $2 \mathrm{D}-\mathrm{hBN}$ and experimental values for $\alpha$-quartz.

\begin{tabular}{lcc}
\hline \hline Formula Unit & $e_{11,2 \mathrm{D}}\left(10^{-10} \mathrm{C} / \mathrm{m}\right)$ & $e_{11,3 \mathrm{D}}\left(\mathrm{C} / \mathrm{m}^{2}\right)$ \\
\hline $\mathrm{C}_{2} \mathrm{HF}(\# 1)$ & 0.630 & 0.188 \\
$\mathrm{C}_{4} \mathrm{HF}(\# 4)$ & 0.079 & 0.017 \\
\hdashline $2 \mathrm{D}-\mathrm{hBN}$ & 1.384 & 0.416 \\
$\alpha$-quartz & --- & 0.171 \\
\hline \hline
\end{tabular}




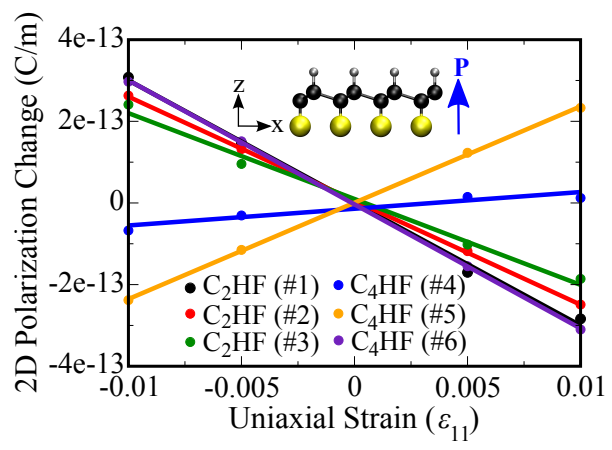

Figure 3. Applying uniaxial strain $\varepsilon_{11}$ along the $x$-direction (defined in top inset) of the graphene sheet induces a change in polarization normal to the plane for each configuration for $\mathrm{C}_{2} \mathrm{HF}$ and $\mathrm{C}_{4} \mathrm{HF}$. Atoms are relaxed under uniaxial strain along the $x$-direction to obtain the relaxed-ion coefficient. The top inset indicates the coordinate system and direction of polarization.

Table 4. Values for the relaxed $e_{31,2 \mathrm{D}}$ piezoelectric coefficients for all configurations of $\mathrm{C}_{2} \mathrm{HF}$ and $\mathrm{C}_{4} \mathrm{HF}$. Values for $e_{31,3 \mathrm{D}}$ were estimated by dividing the $e_{31,2 \mathrm{D}}$ coefficient by the graphene interlayer spacing. These values are compared to the $e_{31}$ coefficient for $\mathrm{Li}$ adsorbed on graphene computed in earlier work. ${ }^{24}$

\begin{tabular}{lcc}
\hline \hline Formula Unit & $e_{31,2 \mathrm{D}}\left(10^{-10} \mathrm{C} / \mathrm{m}\right)$ & $e_{31,3 \mathrm{D}}\left(\mathrm{C} / \mathrm{m}^{2}\right)$ \\
\hline $\mathrm{C}_{2} \mathrm{HF}(\# 1)$ & -0.30 & -0.090 \\
$\mathrm{C}_{2} \mathrm{HF}(\# 2)$ & -0.26 & -0.076 \\
$\mathrm{C}_{2} \mathrm{HF}(\# 3)$ & -0.21 & -0.063 \\
$\mathrm{C}_{4} \mathrm{HF}(\# 4)$ & 0.04 & 0.012 \\
$\mathrm{C}_{4} \mathrm{HF}(\# 5)$ & 0.24 & 0.070 \\
$\mathrm{C}_{4} \mathrm{HF}(\# 6)$ & -0.30 & -0.091 \\
\hline $\mathrm{C}_{2} \mathrm{Li}$ & 0.55 & 0.17 \\
\hline \hline
\end{tabular}


TABLE OF CONTENTS:
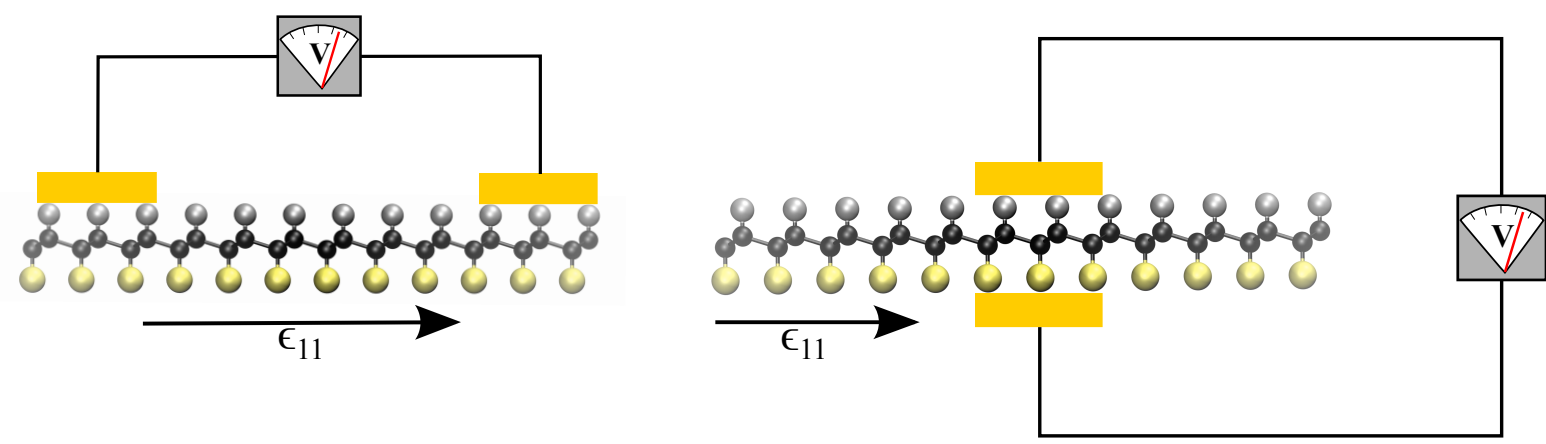